\title{
Influence of Solvent Composition on the Crystal Morphology and Structure of p-Aminobenzoic Acid Crystallized from Mixed Ethanol and Nitromethane Solutions
}

\section{DOI:}

10.1021/acs.cgd.7b00425

\section{Document Version}

Accepted author manuscript

Link to publication record in Manchester Research Explorer

\section{Citation for published version (APA):}

Rosbottom, I., Ma, C. Y., Turner, T. D., O'Connell, R. A., Loughrey, J. J., Sadiq, G., Davey, R. J., \& Roberts, K. J. (2017). Influence of Solvent Composition on the Crystal Morphology and Structure of p-Aminobenzoic Acid Crystallized from Mixed Ethanol and Nitromethane Solutions. Crystal Growth and Design, 17(8), 4151-4161. https://doi.org/10.1021/acs.cgd.7b00425

\section{Published in:}

Crystal Growth and Design

\section{Citing this paper}

Please note that where the full-text provided on Manchester Research Explorer is the Author Accepted Manuscript or Proof version this may differ from the final Published version. If citing, it is advised that you check and use the publisher's definitive version.

\section{General rights}

Copyright and moral rights for the publications made accessible in the Research Explorer are retained by the authors and/or other copyright owners and it is a condition of accessing publications that users recognise and abide by the legal requirements associated with these rights.

\section{Takedown policy}

If you believe that this document breaches copyright please refer to the University of Manchester's Takedown Procedures [http://man.ac.uk/04Y6Bo] or contact uml.scholarlycommunications@manchester.ac.uk providing relevant details, so we can investigate your claim.

\section{OPEN ACCESS}


1 Influence of Solvent Composition on the Crystal Morphology and Structure of $p$ 2 Aminobenzoic Acid Crystallised from Mixed Ethanol and Nitromethane Solutions ${ }^{\mathbf{o}}$

Institute of Particle Science and Engineering and Institute of Process and Research Development, School of Chemical and Process Engineering, University of Leeds, Leeds LS2 9JT, UK

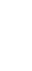

(1) (1)

School of Chemical Engineering and Analytical Science, University of Manchester, Manchester M13 School of Chemistry, University of Leeds, LS2 9JT

${ }^{\circ}$ Paper first presented at the $12^{\text {th }}$ International Workshop on the Crystal Growth of Organic 16 Materials, University of Leeds, UK, June 2016

*Communicating author: i.rosbottom@leeds.ac.uk

+Supplementary material available 


\section{Table of Contents (for review purposes)}

2 Influence of Solvent Composition on the Crystal Morphology and Structure of $p$-Aminobenzoic Acid

3 Crystallised from Mixed Ethanol and Nitromethane Solutions ${ }^{\circ}$ 1

$4 \quad 1 . \quad$ Introduction 5

2. Materials and Methods 6

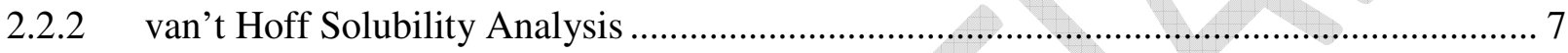

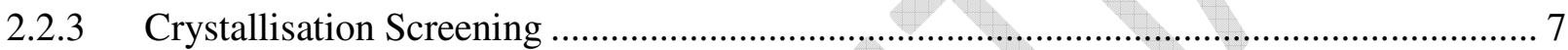

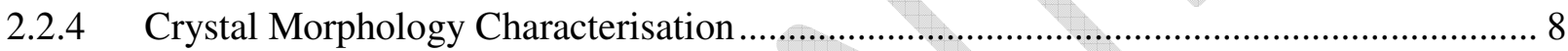

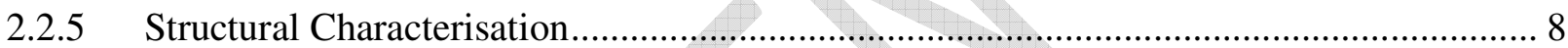

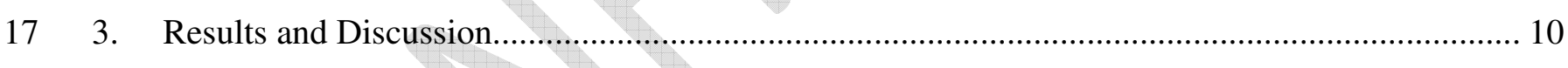

$18 \quad 3.1$ Solvent Selection Application of the Cambridge Structural Database................................. 10

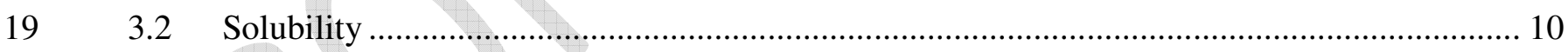

$20 \quad 3.3$ Crystallisation from EtOH:NMe Mixtures................................................................... 12

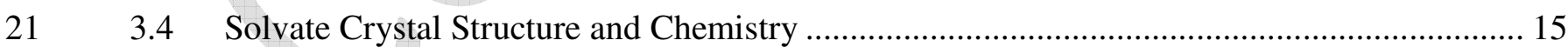

$22 \quad 3.5 \quad$ Crystal Morphologies from Mixed Solvents ….......................................................... 20

$23 \quad 3.6$ Rationalisation of the Morphological and Structural Data through Solute/Solvent Interaction

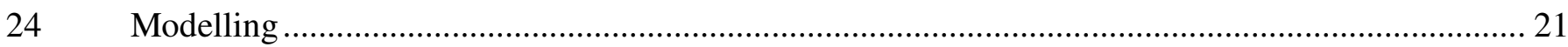


1 Glossary

2 H-bond: Hydrogen bonding

3 vdW: van der Waals

4 XRD: X-ray diffraction

5 PXRD: Powder X-ray diffraction

6 Synthons: Pairwise intermolecular interactions

7 Slice Energy: Energy of intermolecular interactions found within one d-spacing on the $(h k l)$

8 crystallographic plane

9 Attachment Energy: Energy of intermolecular interactions formed when a slice one d-spacing thick is

10 added to a surface defined by $(h k l)$ plane

11 d-spacing: inter-atomic plane separation

12

13 List of Symbols

$14 \Delta H_{f u s}:$ Enthalpy of fusion

$15 E_{c r}$ : Lattice energy

$16 E_{s l}^{h k l}$ : Slice energy per surface $h k l$

$17 E_{\text {att }}^{h k l}$ : Attachment energy per surface $h k l$

18 Å: Angstroms

19 R: Gas constant

20 T: Absolute temperature

$21 \mathrm{~T}_{\mathrm{m}}$ : Melting temperature

$22 \Delta H_{\text {diss }}$ : Enthalpy of dissolution

$23 \Delta S_{\text {diss }}$ : Entropy of dissolution

24

25

26 


\section{Abstract}

2 The crystallographic form and morphology, resulting from the re-crystallisation of $\alpha$-para

3 aminobenzoic acid ( $p \mathrm{ABA})$ from mixed solutions of ethanol (EtOH) and nitromethane (NMe), is

4 reported. From solutions with compositions $>60$ wt $\%$ NMe the $\alpha$-polymorph of pABA appears. In

5 contrast, crystals prepared from mixed solvent with $<60 \mathrm{wt} \%$ NMe reveal the presence of a previously

6 unknown NMe solvate, which crystallises concomitantly with the $\alpha$-structure, whose structure and 7 chemistry are presented here.

8 The NMe solvate crystal structure has similarities to $\alpha-p \mathrm{ABA}$, in particular the existence of -OH...O-

9 H-bonded dimers and $\mathrm{NH} . . . \mathrm{O}$ H-bonds between $p$ ABA molecules, though the $\pi-\pi$ stacking interactions

10 between the phenyl ring groups are found to be much more offset and do not form a continuous chain

11 through the structure, as found in $\alpha-p \mathrm{ABA}$. The crystal structure is refined with a disordered $\mathrm{NMe}$

12 molecule, with the hydrogen atoms, the nitrogen atom and one of the oxygen atoms being found in two

13 occupancies. The $\mathrm{NMe}$ is fond to primarily interact with the $\mathrm{NH}_{2}$ group of $p \mathrm{ABA}$. Intermolecular

14 interactions within the solvate structure are generally weaker than those found in $\alpha-p \mathrm{ABA}$ and the

15 lattice energy is calculated to be significantly lower than $\alpha-p \mathrm{ABA}$. This suggests that the solvate

16 structure is metastable with respect to $\alpha-p \mathrm{ABA}$, and consistent with the observation that pure samples

17 of $\alpha-p \mathrm{ABA}$ are crystallised from solutions with $>60 \%$ NMe content, which have longer induction times

18 and hence produce the more stable $\alpha$-form.

19 The morphology of $\alpha-p \mathrm{ABA}$ crystals produced from solutions with increasing NMe content are found 20 to decrease in aspect ratio, in comparison to needle/lathe shaped crystals produced from pure EtOH. 21 Molecular modelling of the interactions between NMe, pABA molecules and crystal surfaces of $\alpha$ 22 pABA show that $\mathrm{NMe}$ has a greater propensity to interact with the $\mathrm{NH}_{2}$ group and phenyl ring, in 23 comparison to the same calculations for $\mathrm{EtOH}$, which indicate that it prefers to interact with the $\mathrm{COOH}$ 24 group. The data is consistent with NMe disrupting the $\pi-\pi$ stacking interactions that dominate growth 25 along the needle (b-axis) direction of $\alpha$-pABA, resulting in a more isotropic morphology. The 26 propensity of $\mathrm{NMe}$ to interact in this manner is also consistent with the formation of the solvate 27 structure, since $\mathrm{NMe}$ interacts with the slightly less tightly bound and coordinated $\mathrm{NH}_{2}$ and phenyl 28 functional groups in $\alpha-p \mathrm{ABA}$, in comparison to the $\mathrm{COOH}$ group which is more tightly bound to 29 surrounding $p \mathrm{ABA}$ molecules.

30 The correlations between the structural features of $\alpha-p \mathrm{ABA}$ and the solvate, the molecular and surface 31 modelling and the experimental data provide a valuable insight into the effect of crystallisation solvent 
1 on solvate formation and crystal morphology. This is highly relevant to the digital design of a

2 crystallisation process that can aid in the production of highly pure crystalline particles with tailored

3 physical properties.

\section{Introduction}

5 The demand for active pharmaceutical ingredients (API) to be crystallised with high purity and

6 desirable physical properties for downstream unit processes, has resulted in increased importance being

7 attributed to the crystallisation solvent selection process. This is in order to quantify the impact of

8 changes in solvent composition on the physical characteristics of the crystalline product, such as

9 propensity to form solvates ${ }^{1-5}$ and variations in crystal morphology ${ }^{6-13}$. Since a third of materials are

10 known to produce solvates ${ }^{14}$, a detailed understanding of polymorphism and solvate formation can be

11 vital in terms of consistent production of pure and stable drug products. As a caveat to this, the

12 avoidance of needle-like morphologies is also important in pharmaceutical and fine chemical product

13 processing, since they can create problems in downstream processing with their propensity to block

14 filters and pipes, along with their undesirable mechanical properties that can result in their breakage

15 during filtration ${ }^{15}$. Therefore, there is a need to correlate these material properties with molecular and

16 crystal structural packing features ${ }^{1,2,16-18}$, to design highly pure crystalline particles with pre-defined

17 physical properties.

18 Solvent screening can be a key step to assess the crystal form and morphology of APIs produced from

19 solution $^{19}$, in order to ensure the stability of a drug product and avoid product failure, resulting in the

20 expensive process of product withdrawal and re-formulation ${ }^{20}$. Experimental screening can be

21 expensive and time-consuming, hence computational modelling can be used to guide the solvent

22 selection process for API crystallisation ${ }^{2,4,5,17,21-29}$. Once a stable solid form has been confirmed, the

23 crystallisation of this form with isotropic crystal morphology can often be desirable. Though the

24 crystallisation of more isotropic morphologies can sometimes be achieved through control of the solute 25 concentration (supersaturation) during solution crystallisation ${ }^{30-32}$, it can also be achieved by 26 manipulating the solvent composition ${ }^{33-35}$. Previous studies have demonstrated that an understanding of

27 the individual crystal surface chemistries, solvent physical properties and solution supersaturation can

28 result in more effective solvent or additive selection/design for tailoring the crystal growth and

29 morphology $y^{36-39}$. Therefore, the ability to design crystallisation processes via solvent selection and/or

30 other control techniques is of significant importance to produce a stable solid form and desirable

31 crystalline physical properties. 
1 Crystallising from a mixture of solvents with different properties can effectively alter the physical 2 properties (morphology, purity, crystallinity etc.) of a material ${ }^{34,35,40}$. Cook et $a l^{41}$ observed that the mixture of a strong hydrogen bond (H-bond) donor and a strong H-bond acceptor can cause the 4 deviation of the solution properties from regular solution theory ${ }^{42}$. Additionally, Chen et $a l^{9}$ demonstrated the applicability of using a computational approach to the design of a mixed solvation environment that modified the needle-like morphology of 2,6-dihydroxy-benzoic acid. Such studies have identified that understanding a solution crystallisation environment on the molecular level can aide in rationalising the solution behaviour and physical properties of the crystals produced.

9 Drawing upon the above ideas, this study addresses the issue of solvent selection in the crystallisation 10 of $p$-amino benzoic acid ( $p \mathrm{ABA})$, demonstrating firstly how the Cambridge Structural Database (CSD) 11 may be used as a tool for directing solvent choice, and secondly how state of the art synthonic 12 engineering tools ${ }^{17,43,44}$ may be used to aid our understanding of how solvents control both the 13 morphology and phase of resulting solid forms. It is now well known that the $\alpha$ and $\beta$ polymorphs of $14 p \mathrm{ABA}$ are enantiotropically related, having a transition temperature of $\sim 14{ }^{\circ} \mathrm{C}^{45,46}$. It has also been 15 reported that only needle-like crystals of the high temperature stable $\alpha$ form appear from organic 16 solvents (eg alcohols, ethyl acetate, acetonitrile) regardless of the temperature ${ }^{47,48}$. The more prismatic $17 \beta$ pABA only crystallises from water ${ }^{17,47,49,50}$. The search for a new solvent was thus initially 18 motivated by the desire to crystallise the $\beta$ polymorph directly from an organic solvent. As described 19 below, this search led to the use of nitromethane (NMe) and nitromethane/ethanol mixtures as the 20 crystallisation media $^{49}$ and ultimately to examine the solvent effect on crystalline phase and 21 morphology. The results are rationalised using molecular modelling of the solvent interaction with both $22 p \mathrm{ABA}$ molecules and crystal surfaces of $\alpha-p \mathrm{ABA}$ to attempt to understand, at the molecular level, the 23 observed crystalline structural form and morphology produced from the different solvent mixtures.

\section{Materials and Methods}

\subsection{Materials}

Ethanol was purchased from VWR chemicals having a purity of 99.9\% (CAS: 64-17-5). Nitromethane with $>99 \%$ purity was purchased from ACROS Organics (CAS: 75-52-5). The $p$ ABA was purchased from Sigma Aldrich with 99\% purity (CAS: 150-13-0).

\subsection{Experimental Methods}

\subsubsection{Solubility Determination}


1 Saturated solutions of $\alpha-p \mathrm{ABA}$ in pure $\mathrm{EtOH}$, and EtOH:NMe mixtures (NMe concentration was increased in $10 \mathrm{wt} \%$ steps all the way up to pure $\mathrm{NMe}$ ) were prepared at the $10 \mathrm{ml}$ scale, agitated at the desired temperature for 24 hours and allowed to settle for 12 hours, when the supernatant was pipetted into a fresh sample vial and dried. The mass of solid was weighed using a 4-figure balance and the mass checked over 3 days to ensure all solvent had evaporated.

\subsection{2 van't Hoff Solubility Analysis}

7 If a solution is assumed to be ideal, that is the solute/solute, solute/solvent and solvent/solvent

8 interactions are all equal, then the ideal solid/liquid equilibrium can be expressed, assuming a

9 negligible contribution from the heat capacity, as Equation (1)

$10 \quad \ln (x)=\frac{\Delta H_{\text {fus }}}{R}\left[\frac{1}{T}-\frac{1}{T_{m}}\right]$

11 where $R$ is the ideal gas constant, $\Delta H_{\text {fus }}$ is the enthalpy of fusion, $T$ is the temperature and $T_{m}$ is the 12 melting temperature of $\alpha-p \mathrm{ABA}^{51}$. However, since most solutions are non-ideal, the van't Hoff 13 equation can be used to assess the degree of non-ideality, shown in Equation (2)

$14 \ln (x)=-\frac{\Delta H_{\text {diss }}}{R T}+\frac{\Delta S_{\text {diss }}}{R}$

15 where $\Delta H_{\text {diss }}$ and $\Delta S_{\text {diss }}$ are the enthalpy and entropy of dissolution, respectively. Since $R, T, \Delta H_{\text {diss }}$ and $\Delta S_{\text {diss }}$ are constants, the solubility can be plotted as $\ln (x)$ vs $\frac{1}{T}$. Solubility plotted in van't Hoff

17 coordinates that is found to be above the ideal line implies that the solute/solvent interactions are 18 stronger than the solvent/solvent interactions. Conversely, if the solubility is found to be below the 19 ideal line, this then implies that the solvent/solvent interactions are stronger than the solute/solvent 20 interactions. A more in-depth derivation and analysis of this theory can be found in a previous 21 publication $^{52}$.

\subsubsection{Crystallisation Screening}

23 The crystals were obtained from cooling crystallisation in EtOH:NMe solvent mixtures of 0\% (by 24 weight) to $100 \%$ NMe content, at two supersaturations of $S=1.1$ and $S=1.3 .10 \mathrm{~g}$ of solvent with the appropriate amount $\alpha-p \mathrm{ABA}$ were agitated at $50^{\circ} \mathrm{C}$ using a Julabo ${ }^{53} \mathrm{~F} 25$ circulator for an hour to ensure full dissolution of the solid. Then the agitation was halted and the solutions cooled to $10^{\circ} \mathrm{C}$ and left to crystallise, whereby crystallisation occurred within 5 days. Solutions were vacuum filtered to isolate the solid crystalline form from the solution. 
1 Crystals of the pure nitromethane solvate were prepared using slow solvent evaporation. $5 \mathrm{ml}$ of pure

2 nitromethane was saturated with $\alpha-p \mathrm{ABA}$ at ambient temperature and pressure, this was then filtered into a clean $10 \mathrm{ml}$ screw cap vial and covered using plastic paraffin film with a single hole punctured through using a clean needle. The solvent was allowed to evaporate slowly over 3 days; the resulting crystals were isolated using vacuum filtration and air dried.

\subsubsection{Crystal Morphology Characterisation}

7 The crystals obtained were imaged by high quality photography using an Olympus Style TG4 camera.

\subsubsection{Structural Characterisation}

9 Powder X-ray diffraction (PXRD) of the samples was carried out using a Bruker D8 advanced X-ray 10 diffractometer, which used $\mathrm{Cu} \mathrm{K \alpha}$ radiation and a germanium primary monochromator in a Bragg11 Brentano reflection geometry. The step size used was $0.0332 \theta$, with a step time of $180 \mathrm{~s} / \mathrm{step}$ over a $2 \theta$ 12 range of $5-39.8^{\circ} 2 \theta$. The detector used was a Vantec-1 position sensitive detector. Samples were 13 prepared by grinding crystallisation products in a mortar and pestle and mounting on a flat plate sample 14 holder. Data reduction and Rietveld analysis were performed using PANalytical Highscore plus 15 software ${ }^{54}$.

16 For single X-ray diffraction, crystals were immersed in inert oil and mounted on a nylon loop. These 17 were then cooled to $100 \mathrm{~K}$ using an Oxford Cryosystems 700 Series Crysostream Cooler ${ }^{55}$ and data 18 collected on a RigakuSuperNova diffractometer fitted with an Atlas CCD detector with 19 monochromated Mo-K $\alpha$ radiation $(\lambda=0.71073 \AA)$. The dataset was collected and processed using 20 CrysAlisPRO $^{56}$ and corrected for absorption using an analytical numeric correction ${ }^{57}$; the structure 21 solved using SHELXS ${ }^{58}$ and refined by full-matrix least squares on $\mathrm{F}^{2}$ using ShelXL-2014 ${ }^{59}$ interfaced 22 through the programme Olex $2^{60}$. Molecular graphics and tables of data were produced using Olex $2^{60}$. 23 All non-hydrogen atoms were located in the Fourier Map and refined anisotropically. All hydrogen 24 atoms bound to carbon were placed in calculated positions and refined isotropically using a "riding 25 model".

\subsection{Computational Methods}

\subsubsection{Overlaying of Molecular Structures}

28 The molecular structures were overlaid using the 3-point fit module with Interchem $2013^{61,62}$.

\subsubsection{Synthon Characterisation}


1 The strength and nature of the intermolecular interactions in the bulk crystal structure (intrinsic 2 synthons) were characterised using HABIT98 ${ }^{63}$. A nest of unit cells was constructed in three 3 dimensions, and the interactions between a molecule in the central unit cell and all of the other 4 molecules in the surrounding unit cells was calculated. The atom-atom parameters published by 5 Momany et $\mathrm{al}^{64}$ together with fractional charges calculated using the AM1 method within MOPAC ${ }^{65,66}$

6 were used to calculate the strength of the intermolecular interactions in this study. This method for

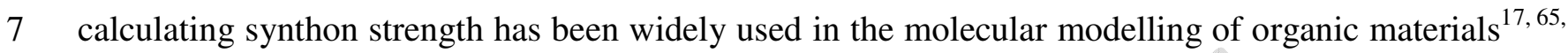
$67-70$

\subsubsection{Molecular and Surface Grid-Based Searching}

10 The most favoured binding sites between the solute and solvents were identified using the systematic 11 search program ${ }^{71-73}$. The program operates with a stationary and mobile phase, in this case they can be 12 single molecules or dimers so will simply be referred to as a 'body'. The stationary body is fixed, 13 whilst the mobile body moves around the stationary body on a grid. At each grid point, the mobile 14 phase can also rotate about three angles. At each point and rotation, the intermolecular energy was 15 calculated using the same potential parameters mentioned in Section 2.3.2.

16 In this study, the most favoured binding sites of up to 5 molecules of NMe and ethanol with a $p \mathrm{ABA}$ 17 dimer were identified. The binding sites were searched sequentially. For the first simulation, the $p \mathrm{ABA}$ 18 dimer was held rigid and the solvent molecule was moved around the grid surrounding the dimer and 19 the most favourable interaction between a solvent molecule and the dimer was identified. The 20 coordinates for the most favoured interaction between the $p \mathrm{ABA}$ dimer and one solvent molecule was 21 used as the starting point for the second simulation, where it was held rigid and a second solvent 22 molecule was searched in the same way as the first simulation to find the most favoured binding site of 23 a second solvent molecule. This search procedure was repeated for up to 5 molecules of the solvent.

24 For the surface searching ${ }^{74,75}$, a crystal surface, represented by a slab, was generated and the slab was then treated as a fixed body during searching process. The thickness of the slab was selected to be a multiple of the $\mathrm{d}$-spacing $\left(\mathrm{d}_{h k l}\right)$ between adjacent $(h k l)$ lattice planes, whilst the surface area was a

28 eliminate the edge effects on the search results. The grid cells were generated based on three orientations of the mobile body and two spherical polar angles between the two bodies, but only 30 covering half of the space facing the crystal surface, i.e., the azimuthal angle from 0 to $180^{\circ}$. The 31 search explored every grid point and calculated the interaction energy between the slab and the mobile 
1 body (a probe molecule or cluster), and hence identify the most favourable sites for the mobile body to

2 dock on or interact with the slab surface.

\section{Results and Discussion}

\subsection{Solvent Selection Application of the Cambridge Structural Database}

6 A starting point in this study was a search of the CSD, with the objective of identifying molecular

7 functionalities that could preferentially interact with the acid group of $p \mathrm{ABA}$. Accordingly the CSD

8 was searched for all crystal structures containing the pABA moiety. Excluding the 6 entries for pure

$9 \quad p \mathrm{ABA}$ and the structures in which $p \mathrm{ABA}$ adopted the acid dimer, this amounted to 42 hits in which the 10 acid hydroxyl was bound to a non $p$ ABA group. Of these, 29 involved H-bonds directly to a nitrogen 11 (as pyridine, bipyridine, imidazole) and 13 were bound to oxygen either as carbonyl (6), water (2) or 12 nitro (5) functionality. On this basis, it was surmised that nitromethane would be an interesting solvent 13 to explore since it had not previously been chosen for crystallisation experiments. To our knowledge 14 this is the first report of the application of the CSD in solvent selection.

\subsection{Solubility}

16 Figure 1 shows the solubility measured in pure $\mathrm{EtOH}, \mathrm{NMe}$, and in mixtures of EtOH/ NMe in steps of $10 \%$ by weight in the temperature range $10-30^{\circ} \mathrm{C}$. 


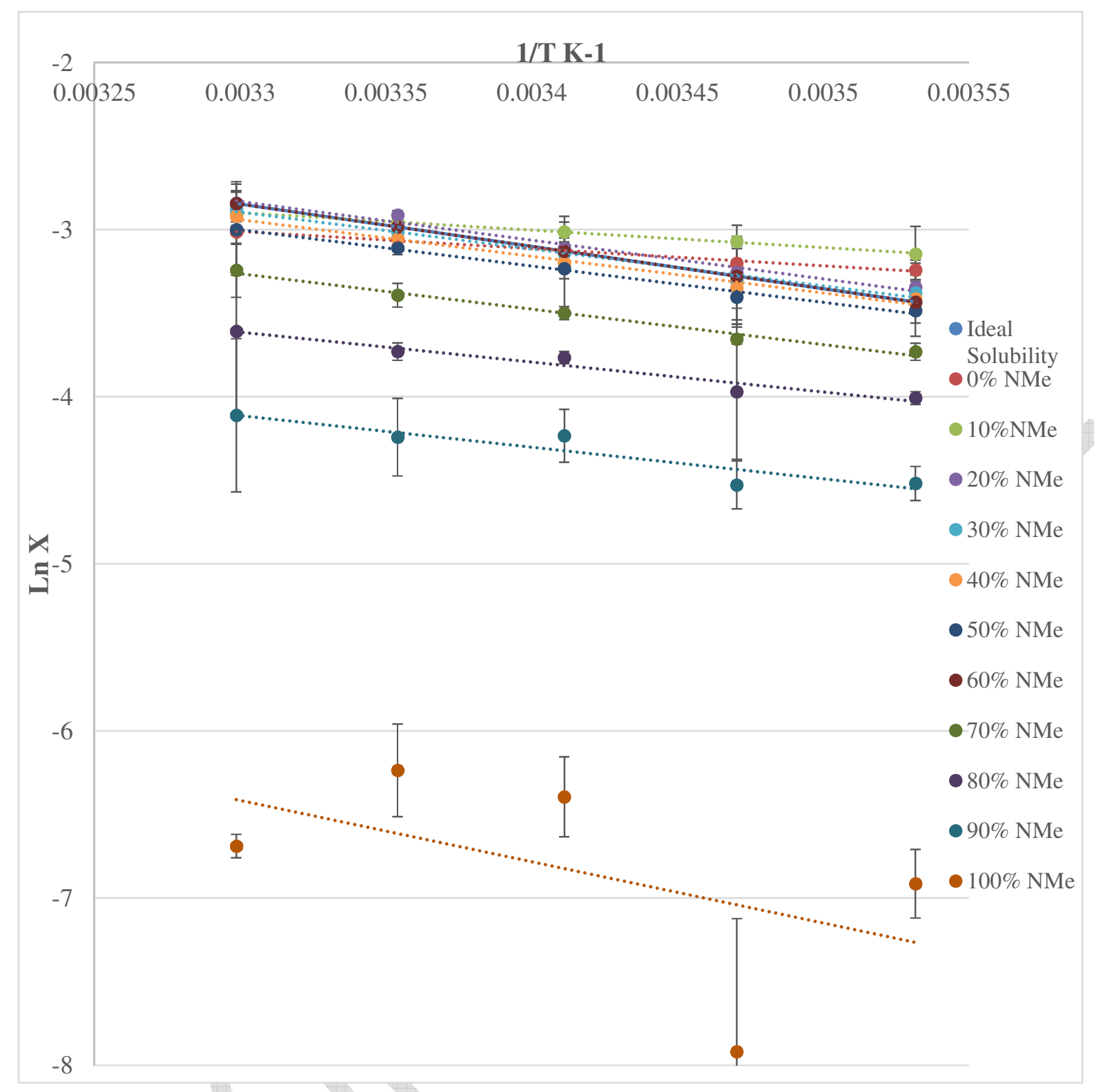

Figure 1: The solubility for the different solvent compositions plotted using the van't Hoff relationship with the ideal solubility (equation 1) indicated by the solid black line;

6 The data in Figure 1(b) shows that only the solubility up to about 30\% NMe content was above the 7 ideal solubility line, consistent with the increase in solubility seen upon addition of small amounts of $8 \mathrm{NMe}$ (Figure S1(a), supplementary material). It was also observed that the solubility at lower 9 temperatures was further above the ideal solubility than at higher temperatures, which suggests that 10 these liquids may be able to be significantly undercooled and remain stable ${ }^{52}$. The large errors observed 11 for the van't Hoff coordinates for pure NMe were due to the small amount of material produced from 
1 the solubility experiments, hence when measuring the mass of the material, any small deviation in the

2 mass was translated into a large percentage error.

\subsection{Crystallisation from EtOH:NMe Mixtures}

5 Cooling crystallisation experiments yielded crystalline particles from all the solutions except pure

6 nitromethane, where after 5 days no crystalline material could be obtained. The comparison of the 7 PXRD spectra of the crystals produced at $10^{\circ} \mathrm{C}$ from the solutions containing $10 \%, 30 \%, 50 \%$ and $80 \%$ $8 \quad$ NMe are shown in Figure 2.

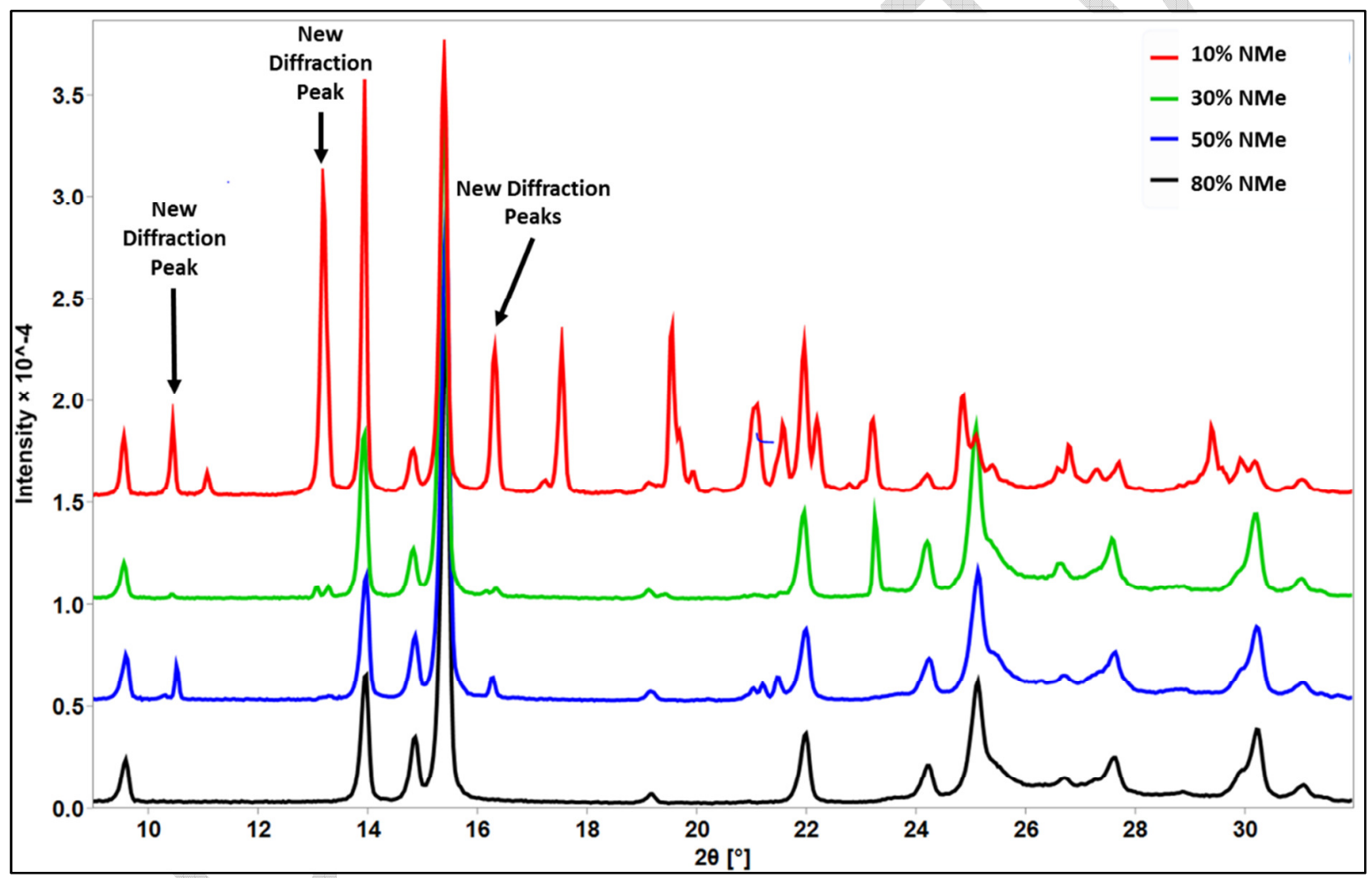

11 Figure 2: Comparison of the PXRD spectra of the crystals produced from the solution containing $1210 \%$ NMe (red); $30 \%$ NMe (green); $50 \%$ NMe (blue) and $80 \%$ NMe (black). New peaks labelled 13 are peaks not consistent with $\alpha-p$ ABA structure

15 The crystals produced from the 90:10 EtOH:NMe sample displayed peaks that were consistent with the $16 \alpha-p$ ABA pattern, along with some prominent peaks not present in the $\alpha-p$ ABA pattern (labelled 'new 17 diffraction peaks' in Figure 2). These previously unseen peaks, consistent with the crystallisation of a 
1 mixture of phases, were also observed in the 30:70 and 50:50 diffraction patterns, albeit at much lower

2 intensities than in the 90:10 pattern. However, the 20:80 pattern showed no evidence of these

3 previously unseen peaks.

4 A single crystal formed from cooling crystallisation of a highly supersaturated solution of pure NMe 5 was isolated and subject to single crystal XRD. This revealed (see section 3.4) that a $p \mathrm{ABA} \mathrm{NMe}$ 6 solvate structure had been formed. The calculated PXRD pattern based on this solvate was consistent 7 with, and hence explained, the previously unseen peaks found in some of the PXRD patterns from the 8 mixed solutions (Figure 2). Figure 3 shows the comparison of calculated PXRD patterns of the NMe 9 solvate and $\alpha-p$ ABA structures, with the PXRD patterns from the crystals produced from solutions 10 containing $10 \%, 20 \%, 30 \%$ and $50 \%$ NMe content.

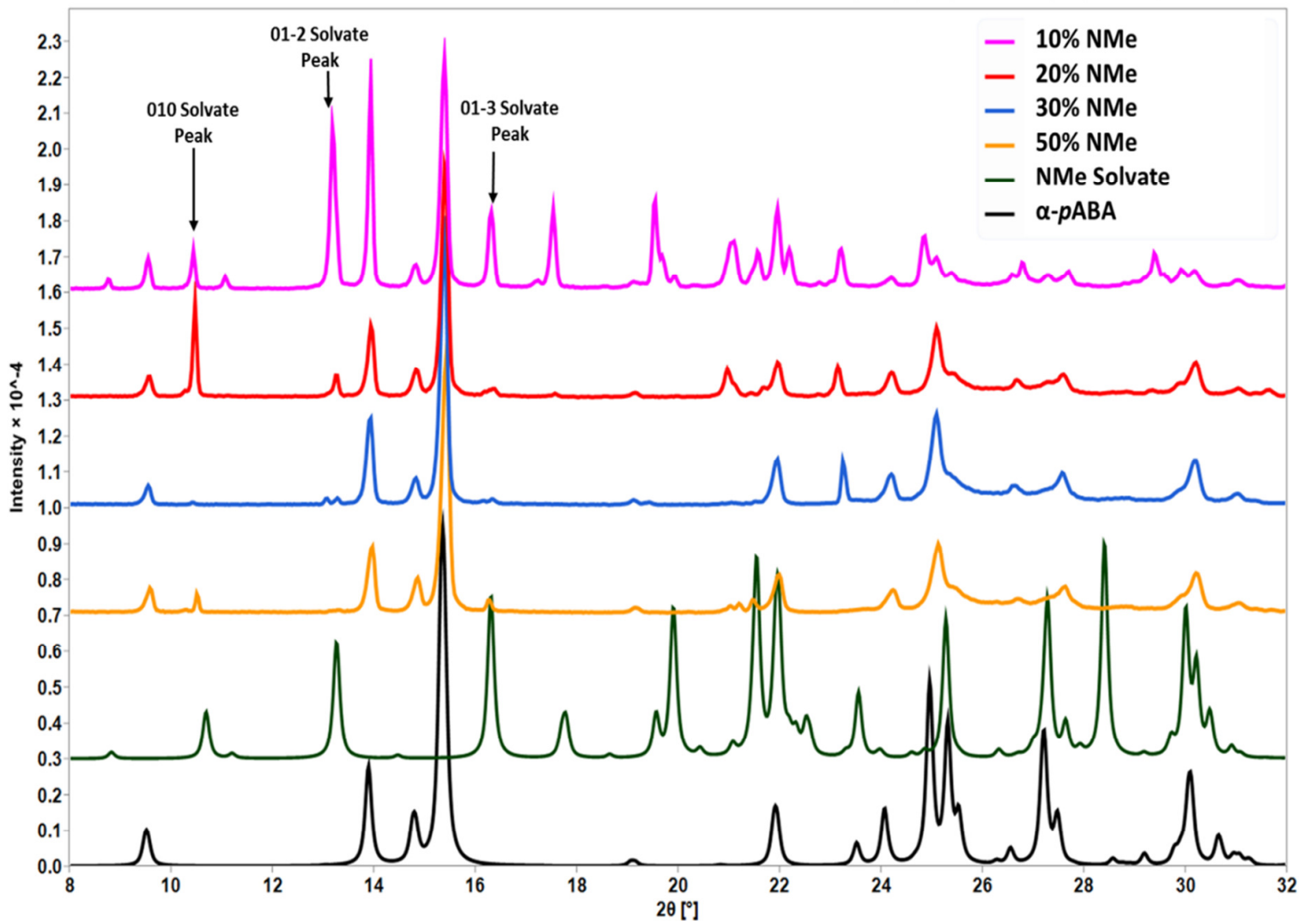

Figure 3: Overlay of experimental diffraction patterns for the 10, 20, 30 and $50 \%$ NMe samples, 13 which contained peaks related to the solvate phase, with a comparison to the simulated 14 diffraction patterns of the NMe solvate and $\alpha-p$ ABA structure files 
1 Figure 3 shows that the 'new peaks' from Figure 2 can be attributed to the 010,01-2 and 01-3 peaks of

2 the solvate crystal structure. The quantity of the solvate in each sample was calculated by full pattern

3 Rietveld analysis, with pattern fitting to both the $\alpha-p \mathrm{ABA}$ and the solvate structure files.

4 Table 1: Weight percentage values of the solvate in the samples containing solvate peaks, 5 together with the goodness of fit of the Rietveld refinement. *Solution did not crystallise over 5 6 days using the method described in section 2.2.3, hence the production of the pure NMe solid was 7 achieved using slow solvent evaporation in pure NMe

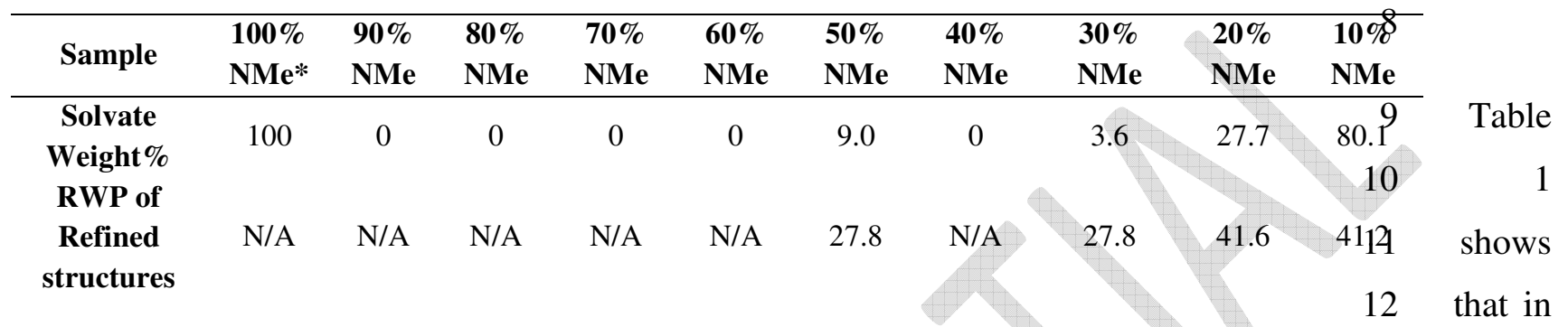

13 general, the amount of solvate present in the samples is reduced with increasing NMe content, with a 14 maximum $80.1 \%$ of the solvate in the $10 \%$ NMe sample. It was observed that the samples with $40 \%$ 15 and 70-90\% NMe content contained no evidence of the solvate structure. It was also observed that the 16 time taken to crystallise the solid increased with increasing NMe content in the solution. Therefore this 17 suggests that the decrease in the amount of solvate structure with increasing NMe content could be 18 consistent with a meta-stablity of the solvate structure, i.e. with increased induction time to 19 crystallisation resulting in the more stable $\alpha-p \mathrm{ABA}$ structure. If the solvate phase was the more stable 20 phase, it would be expected that the increase of NMe content would enhance the crystallisation of the 21 solvate phase, however the fact that the solvent composition would impact on the transition 22 temperature between the two phases must also be considered. From the data collected it seems likely 23 that the $\alpha$-phase is more stable than the solvate phase, and that nitromethane impacts on the relative 24 crystallisation rates of the two forms.

25 However, it should be highlighted that the 100\% NMe sample included in Table 1 was produced from a 26 slow solvent evaporation experiment in pure NMe, which yielded a solid of the pure NMe solvate and a 27 separate solid which was a mixture of $\alpha-p \mathrm{ABA}$ and the NMe solvate. This therefore suggests that it 28 takes extremely careful control over the crystallisation conditions to produce a sample of the pure $\mathrm{NMe}$ 29 solvate structure.

30 It should also be mentioned that for the solubility measurements given in Figure 1, the solid phase that 31 was harvested from the supernatant was assumed to be $\alpha-p \mathrm{ABA}$. However, considering that it has now 32 been shown in Figures 2 and 3, along with Table 1, that a mixture of the $\alpha-p$ ABA and NMe solvate 
1 phases can be obtained from the cooling crystallisation experiments in the EtOH:NMe solvent

2 mixtures. Therefore, it must be stated that the solubility measurements quoted here could be influenced

3 by the conversion of any of the material to the NMe solvate, and that currently the solubility of this

4 form is currently unknown. Hence, a future study that further examines the crystalline phase obtained

5 from the gravimetric analysis solubility measurements, along with identifying the solubility of the

6 solvate phase, could be of interest.

\subsection{Solvate Crystal Structure and Chemistry}

8 The unit cell and packing of the solvate crystal structure is shown in Figure 4.

9
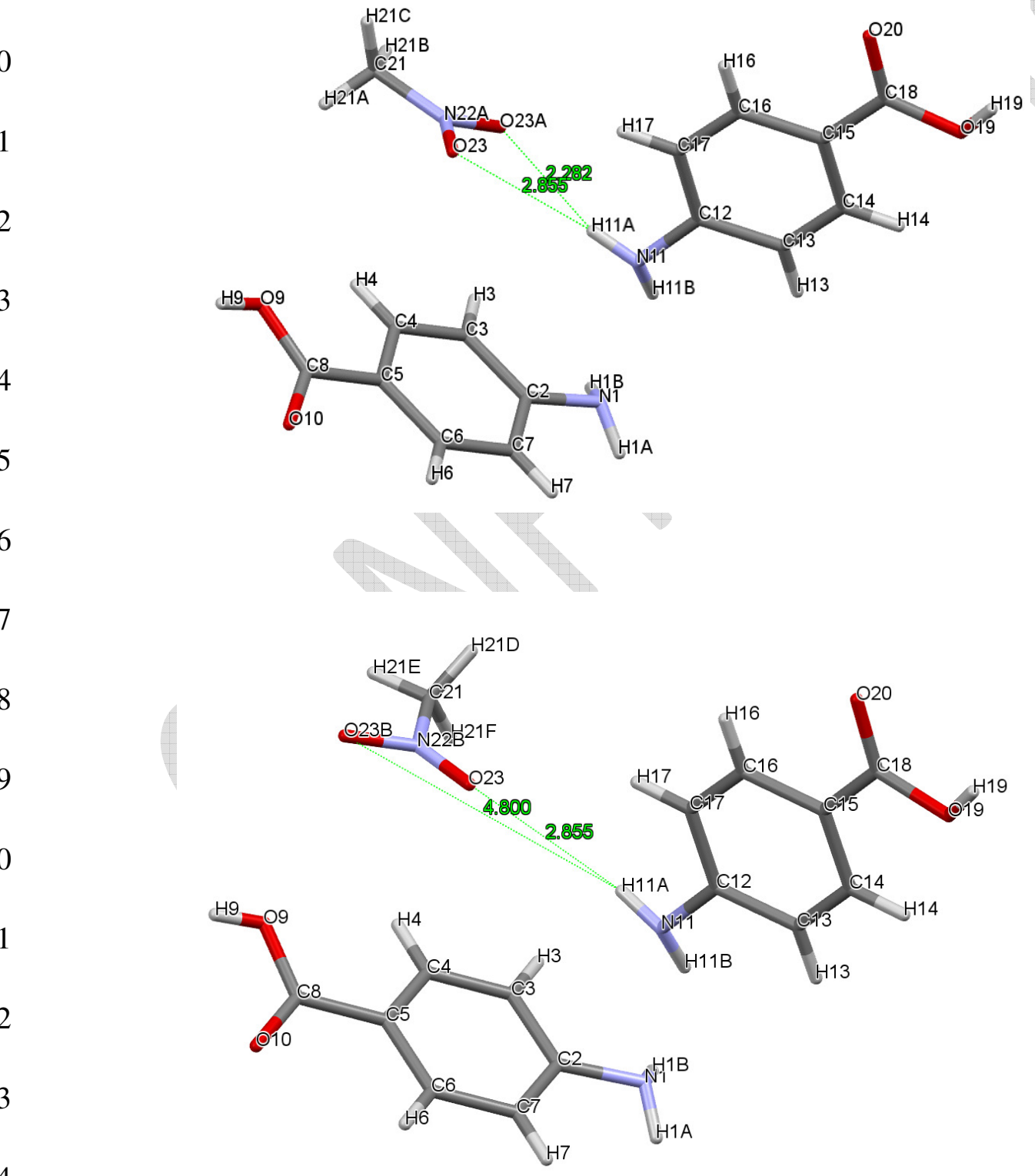


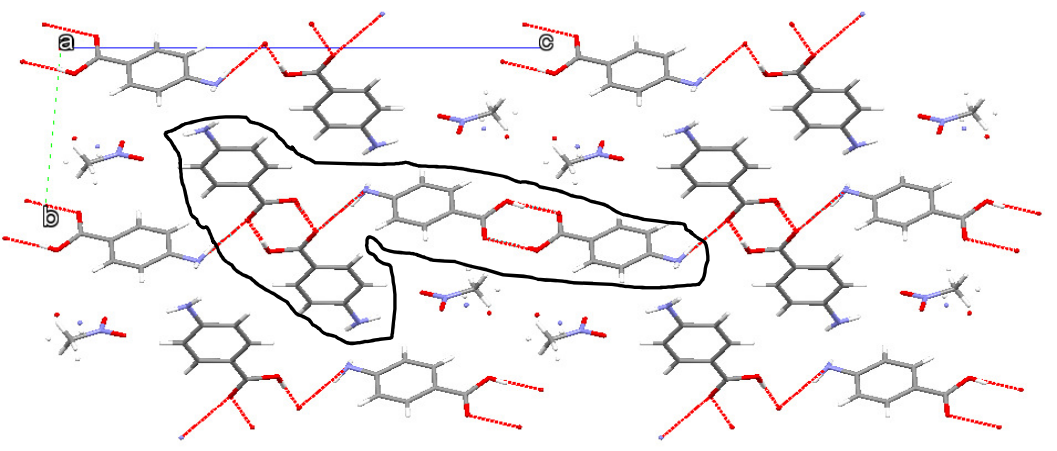

(c)

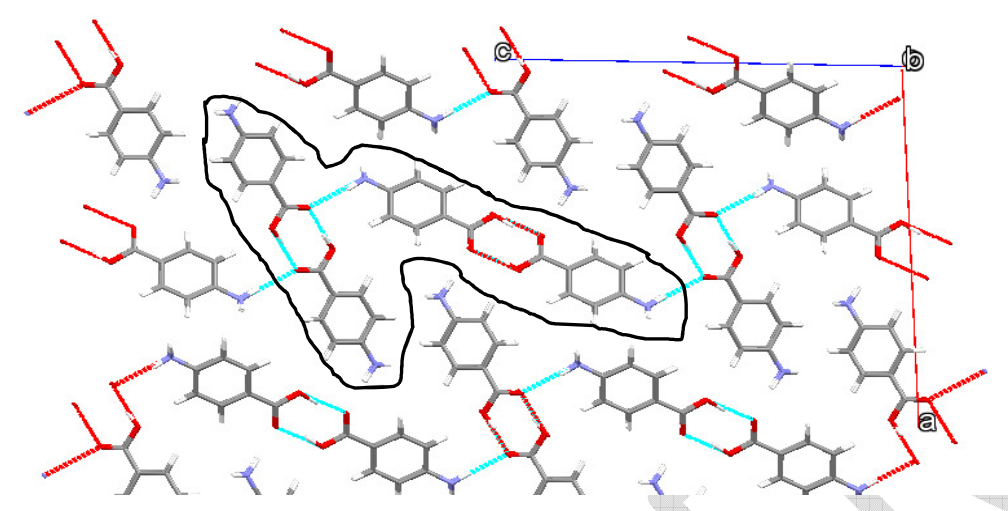

Figure 4: (a) asymmetric unit of occupancy 1 (54\%); (b) asymmetric unit of occupancy 2 (46\%); (c) packing of the NMe solvate structure in the yz plane, black line identifying the tetramer common to the $\alpha-p \mathrm{ABA}$ structure and solvate structure; (d) packing of the $\alpha-p \mathrm{ABA}$ structure in the $\mathrm{xz}$ plane, black line identifying the tetramer common to the $\alpha-p \mathrm{ABA}$ structure and solvate structure.

Figure 4(a) shows that the solvent molecule with 54\% occupancy was found to have both oxygens less than $3 \AA$ away from $\mathrm{H11A}$ on the amino group of $p \mathrm{ABA}$, whilst the NMe molecule was found to be rotated in the $46 \%$ occupancy with $\mathrm{O} 23 \mathrm{~B}$ being found to be $4.8 \AA$ away from H11A

It was observed in the PXRD patterns of the crystals produced from the different solvent mixtures that some peaks were found to shift non-systematically in $2 \theta$ from sample to sample. This was attributed to the disorder of the NMe in the structure, whereby the NMe could occupy the different atomic positions identified from the single crystal structure, or even a mixture of the two (full details in supplementary material).

The black lines in Figure 4 (c) and (d) highlight the existence of essentially identical H-bonded tetramers, (two acid dimers linked by an NH...O hydrogen bond), which exist in both the solvate and $\alpha-p$ ABA structure. This would suggest that, analogous to the $\alpha$-structure, that the $\mathrm{OH}$...O H-bonding dimers play a significant role in stabilising the crystal structure of the solvate. The orientations of the two carboxylic acid H-bonding dimers within the two crystal structures are compared in Figure 5. 
Dimers can stack

3

4

5

6

7

8

9

10 approximately along the b-axis

direction in $\alpha$-structure

(a)

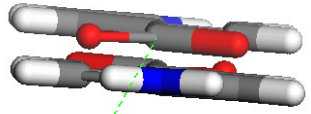

(c)

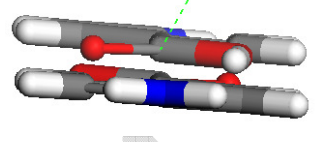

(b)

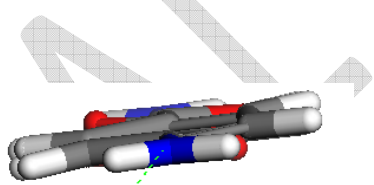

(d)

Dimers are twisted and cannot stack in the same direction in solvate

(1)

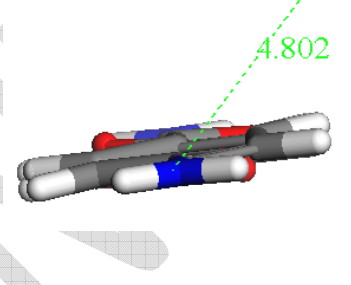

Figure 5: (a) the orientation of the two independent carboxylic H-bonding dimers within the $\alpha$ pABA structure; (b) Orientation of the two independent carboxylic acid H-bonding dimers and the NMe molecule within the NMe solvate structure; (c) the stacking of the carboxylic acid dimers in $\alpha-p \mathrm{ABA}$; (d) stacking of the carboxylic acid $p \mathrm{ABA}$ dimers in the NMe solvate structure

Figure 5(a) shows how the two independent acid dimers pack with the ring structures approximately pointing in the same direction, allowing for efficient close stacking of the dimers through their ring structures along the b-axis ${ }^{17,49}$. Comparing (c) and (d), it was found that the stacking of the $p$ ABA dimers in the solvate structure $((\mathrm{d}))$ were far more offset from each other than in the pure $\alpha-p \mathrm{ABA}$ structure ((c)), and the measured distance between two adjacent carbonyl carbons was found to be approximately $1 \AA$ greater in the solvate structure. It can also be observed that the stacking interactions in the $\alpha$-structure form in a continuous and unbroken chain, approximately in the direction of the baxis. In comparison, the stacking in the NMe solvate structure was found to be isolated interactions, rather than a chain of $\pi-\pi$ stacking.

The $\mathrm{b}$-axis of the solvate structure was found to lengthen in comparison to the $\alpha-p \mathrm{ABA}$ structure. Figure 4(d) shows that the NMe molecule is situated between the rings of two $p$ ABA molecules, hence 
1 forcing the extension along the b-axis. In the $\alpha-p \mathrm{ABA}$ structure, both dimers are approximately in the 2 same plane (Figure 5(a)) and the hydrogen bonds are directed approximately in the A and C directions, 3 hence they are approximately the same in length. In the solvate structure, the twisting of the dimers to 4 accommodate the NMe molecule has resulted in the dimers no longer being in the same plane running 5 along the a-axis, hence the a-axis has reduced (Figure 4(c) and (d)).

6 The calculated strength of the intermolecular interactions (synthons) found within the NMe solvate is 7 shown in Table 2, along with visualisation of the pairwise interactions.

8

9 
Table 2: Strongest synthons calculated from the bulk structure of the NMe solvate structure and compared to the strongest synthons from the $\alpha-p$ ABA structure. Synthons visualised either side of the table for each structure

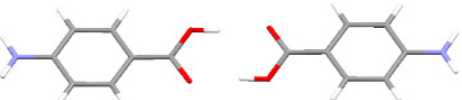

9
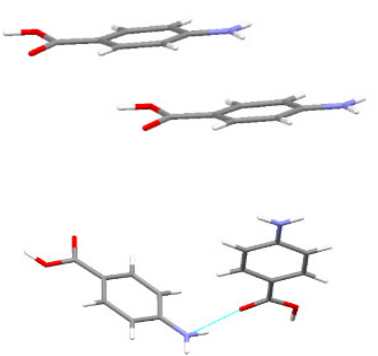
14
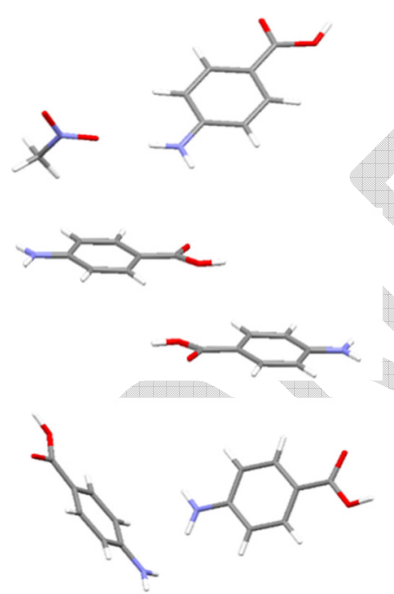

The lattice energy calculated for the solvate structure was found to be almost $7 \mathrm{kcal} / \mathrm{mol}$ less than the

22 lattice energy calculated for $\alpha-p \mathrm{ABA}$. Synthons A, B and C were found to be structurally similar to the

23 strongest synthons found in a study of the $\alpha-p$ ABA crystal structure ${ }^{17}$, however they were in general

24 calculated to be weaker in the NMe solvate structure when compared to their counterparts in the $\alpha$ $25 p$ ABA structure ${ }^{17}$. The significantly reduced calculated lattice energy, weaker synthons and decrease in 26 packing density (Table S2) is consistent with a metastable structure, which correlates to the 
1 crystallisation data in Section 3.3 Some extra hydrogen bonding interactions were also identified, an

$2 \mathrm{NH} . . . \mathrm{O}$ H-bond between the $p \mathrm{ABA}$ and NMe molecules and an NH...N H-bond between the $p \mathrm{ABA}$

3 molecules, which was not found to be present in the $\alpha-p \mathrm{ABA}$ structure ${ }^{17}$. The presence of the extra

4 hydrogen bonds probably somewhat compensates for the general weaker intermolecular synthonic

5 structure, hence stabilising the crystal structure.

\subsection{Crystal Morphologies from Mixed Solvents}

Figure 6 shows images of the crystals produced from the cooling crystallisation experiments.

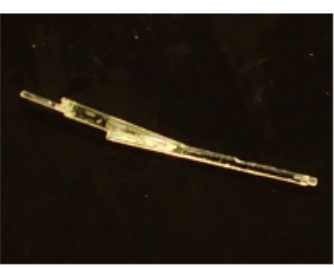

$100 \%$ EtOH

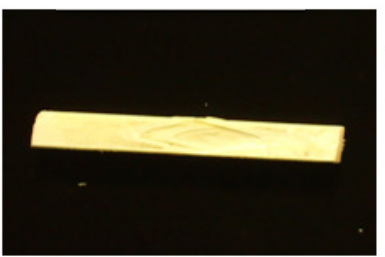

70\%:30\%

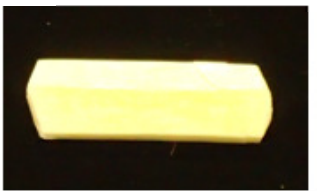

$40 \%: 60 \%$

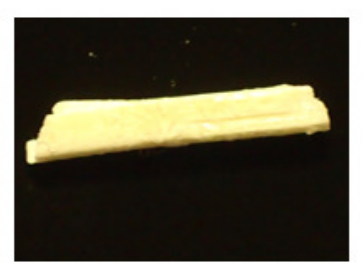

$90 \%: 10 \%$

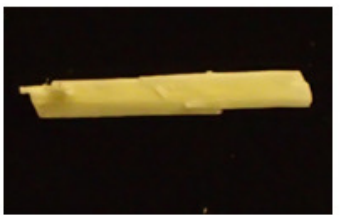

$60 \%: 40 \%$

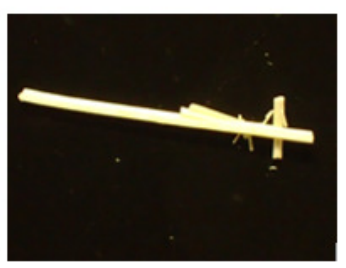

$\mathbf{8 0 \%}: \mathbf{2 0} \%$

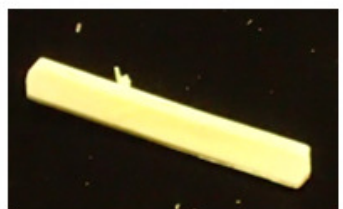

$50 \%: 50 \%$

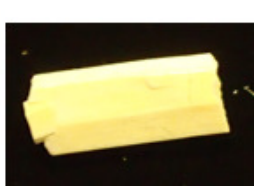

$30 \%: 70 \%$

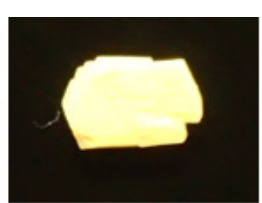

$20 \%: 80 \%$

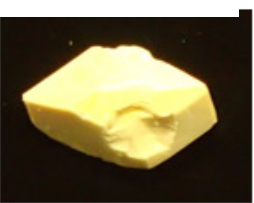

$10 \%: 90 \%$

Figure 6: Images of $p \mathrm{ABA}$ crystallised at $\mathrm{S}=1.1$ at $10^{\circ} \mathrm{C}$ from pure $\mathrm{EtOH}$ up to $90: 10$ NMe:EtOH ratio

Figure 6 shows that the crystals produced from solutions with increasing NMe content were found to decrease in aspect ratio and become more opaque. Table 1 indicated that the crystals produced from solutions with above $60 \%$ NMe content were pure $\alpha$-pABA, therefore suggesting that the increasing NMe content reduces the aspect ratio of pure $\alpha-p$ ABA crystals. Figure 6 also showed that the crystals became more facetted and have smoother surfaces with increasing NMe content. Previous studies suggested that the capping faces which terminate the $b$-axis of $\alpha-p \mathrm{ABA}$ grow by a rough interfacial growth mechanism from ethanol ${ }^{76}$ and that they are often not well formed upon solution 
1 crystallisation $^{49}$. Therefore, addition of NMe to the solutions appears to stabilise the interface and

2 encourage slower, stable growth of the $\alpha$-pABA capping faces.

3

4

8

9

10

11

12

13

14

15

16 

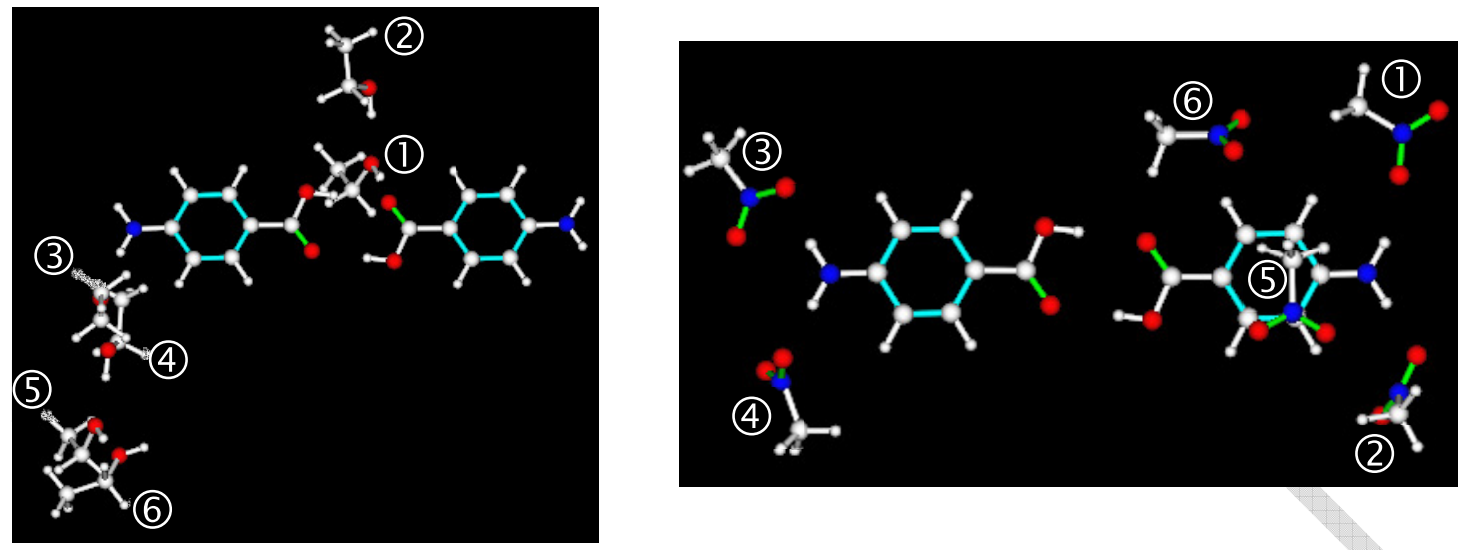

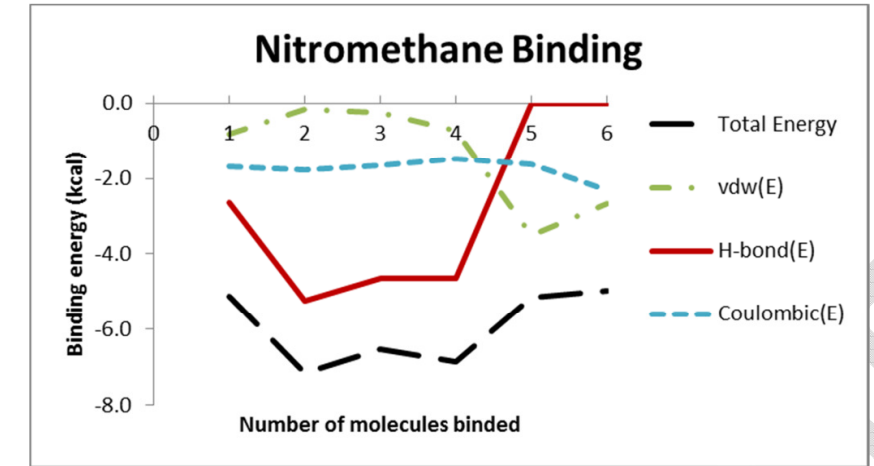

2

3

4

5

6

7

8

3

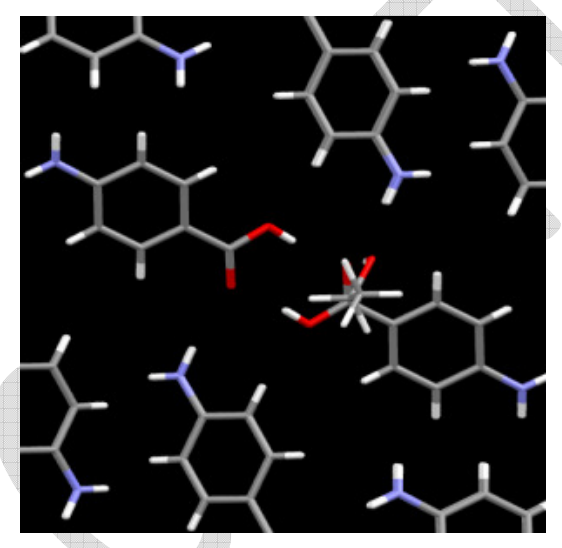

(a)

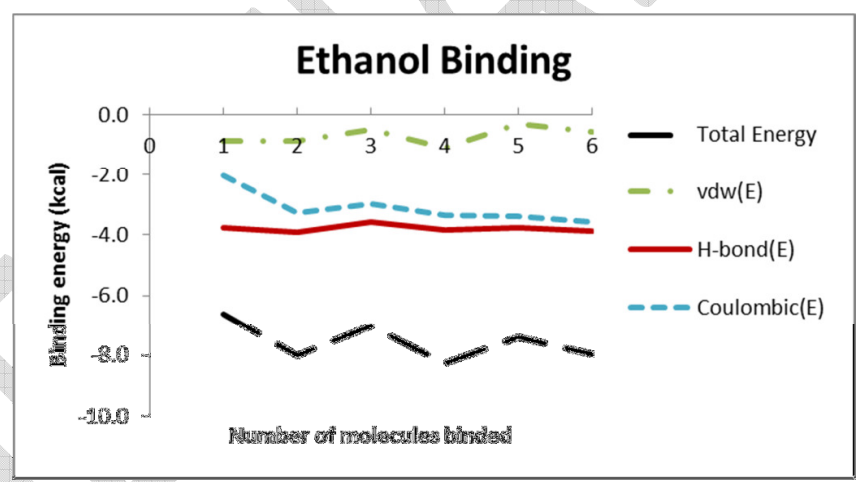

(b)

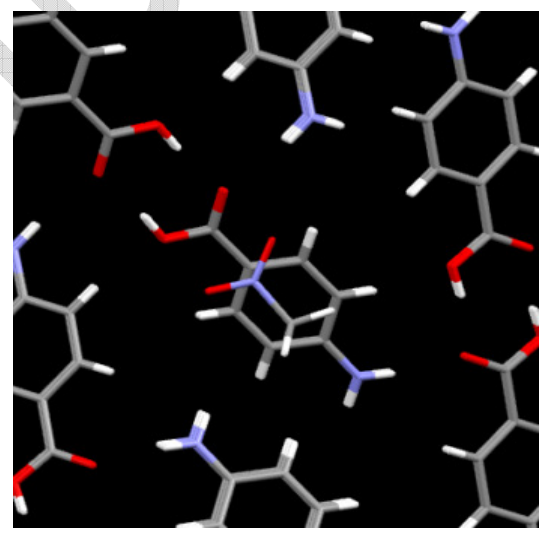

(c)

Figure 7: (a) Comparison of the sequential systematic searching of 6 molecules of ethanol (left) and nitromethane (right); (b) energetic contributions to the sequential molecular binding calculated by the systematic search for ethanol (left) and nitromethane (right) (c) most favoured orientation for a probe molecule of ethanol (left) and nitromethane (right) at the ( 10 -1) surface of the $\alpha-$ form of $p \mathrm{ABA}$. 
1 Figure 7 (a) shows that the EtOH molecules were found to prefer to bind to the $\mathrm{COOH}$ and $\mathrm{NH}_{2}$ 2 groups, whilst the $\mathrm{NMe}$ molecules were found to prefer to bind to the $\mathrm{NH}_{2}$ group and the phenyl ring. 3 This correlates to the orientation of the NMe molecule found within the solvate structure (Figure 4).

4 This observation may be related to the maximum observed in the solubility dependence of $p \mathrm{ABA}$ on 5 NMe content. This would imply that at a level of $10 \% \mathrm{NMe}$ in EtOH both the phenyl ring and the 6 carboxylic acid group of $p$ ABA experience their maximum solvation.

$7 \quad$ Figure 7 (b) revealed that the vdW interactions had a relatively small contribution to the binding energy 8 for the EtOH molecule. However, after the addition of a fifth NMe molecule, the vdW contribution to 9 the energy started to increase strongly, consistent with the NMe molecule having a stronger interaction 10 with the phenyl ring. This is supported by Figure 7(c), which shows that the systematic search 11 suggested that, in its most favourable position, the NMe molecule prefers to lie across the phenyl ring 12 at the capping face of $\alpha-p \mathrm{ABA}$, whilst EtOH adopts a more orthogonal orientation in order to bind most 13 strongly to the $\mathrm{COOH}$ group.

14 It is interesting to comment that, as well as nitromethane, solvate structures have been produced from 15 acetone and dioxane ${ }^{77}$. In contrast, though there have been extensive studies of the crystallisation of 16 ABA from polar protic solvents, such as water and ethanol, no solvate structures have been found and $17 \alpha-p \mathrm{ABA}$ often crystallises ${ }^{45-47,52,78}$. This observation could correlate with the fact that EtOH, which is 18 a polar and protic solvent, was found to prefer to strongly interact with the $\mathrm{COOH}$ group of $p \mathrm{ABA}$. The $19 \mathrm{COOH}$ group forms several strong interactions with other $p \mathrm{ABA}$ molecules in the $\alpha-p \mathrm{ABA}$ structure, 20 implying that there is probably not much space around this group. In contrast, the $\mathrm{NH}_{2}$ and phenyl ring 21 groups probably have a greater amount of space around them in the structure, and the NMe molecule is 22 found to have a greater propensity to interact with those groups. Indeed, the NMe molecule is found to 23 be interacting primarily with the $\mathrm{NH}_{2}$ group in the solvate structure. Hence, this implies that the space 24 around functional groups, along with the solvent/solute interactions with respect to these functional 25 groups, has a strong influence on the propensity of a particular solvent to produce a solvated crystalline 26 form.

27 We also suggest that the solute/solvent interactions in the crystal structure and calculated from the 28 molecular and surface systematic searches have implications with respect to the changes in crystal 29 morphology observed in Section 3.5. It has been observed that at the capping surface of $\alpha-p \mathrm{ABA}$, the $30 p$ ABA molecules integrate into the surface by forming $\pi$ - $\pi$ stacking interactions between the phenyl 31 ring structures ${ }^{17}$. Therefore the observation that the NMe molecule has a greater propensity to interact 
1 with the phenyl ring, in comparison with ethanol, could imply that the presence of NMe results in the

2 observed reduction of the aspect ratio of crystals produced from solutions of increasing $\mathrm{NMe}$ concentration. The surface search results shown in Figure 7(c) suggest that, at the capping face, the binding of the NMe could potentially hinder the formation of the chain of $\pi-\pi$ stacking interactions that are thought to strongly influence the needle-like morphology of $\alpha-p \mathrm{ABA}^{16-18,49}$. This is supported by the observation that such chains of close stacking phenyl rings in the $p \mathrm{ABA}$ molecules, observed in the $\alpha-p \mathrm{ABA}$ structure, were found to be absent in the solvate.

\section{Conclusions}

10 This study has demonstrated for the first time how a combined use of the CSD, crystal structure 11 analysis and molecular and crystal surface modelling of the solute-solvent interactions, can provide an 12 insight into the crystalline form and morphology produced from the different solvent mixtures. The 13 calculated propensity of $\mathrm{NMe}$ to interact more strongly with the $\mathrm{NH}_{2}$ and phenyl ring groups, in 14 comparison to a polar protic solvent like EtOH which was calculated to prefer to interact with the $15 \mathrm{COOH}$ group, probably resulted in the formation of the solvate structure, as there was space around the $16 \mathrm{NH}_{2}$ group for the NMe molecule to be accommodated.

17 The observed disorder of the NMe molecules in the solvate crystal structure accounted for the shifting 18 of the peaks in the PXRD, suggesting that the solvate structure could occupy different occupancies 19 when crystallised from solution. This correlated well to the calculated weaker synthonic interactions in 20 the solvate structure, in comparison to the $\alpha$-structure, along with the observation that $\alpha-p \mathrm{ABA}$ 21 dominated the crystallisation from solutions with $>60 \% \mathrm{NMe}$ content, due to the increased 22 crystallisation time.

23 The greater propensity of the NMe molecule to interact strongly with the phenyl ring group of $p \mathrm{ABA}$, 24 along with the observed disruption in the $\pi-\pi$ stacking interactions in the solvate structure, has been 25 correlated to the decrease in aspect ratio of crystals produced from solutions with increasing NMe 26 content. Since the $\pi-\pi$ stacking interactions dominate the growth along the long axis of the needle, the presence of NMe indicates its disruption to these interactions, hence slowing down the growth along 28 the long axis of the needle, producing the observed isotropic morphologies.

29 This work demonstrates how molecular and crystallographic modelling tools can be combined with in30 depth particle analysis to explain the changes in physical properties of a crystalline material. This work 31 can assist in the on-going efforts to improve the digital design of crystallisation from solution, in 
1 relation to assisting the cleaner and more efficient manufacture of pharmaceutical and fine chemical particulate products.

\section{Acknowledgements}

4 We are grateful to the EPSRC for the support of crystallisation research at Leeds and Manchester through the award of a Critical Mass grant 'Molecules, Clusters and Crystals' (EP/I014446/1), which supported the PhD studies of two of us (I.R ${ }^{79}$ and T.D. ${ }^{80}$ ). We also gratefully acknowledge the support of the Advanced Manufacturing Supply Chain Initiative through the funding of the 'Advanced Digital Design of Pharmaceutical Therapeutics' (Grant No. 14060) project in terms of supporting pharmaceutical crystallisation and modelling research at Leeds. We would also like to thank Dr Christopher Pask (School of Chemistry, University of Leeds) for helpful discussions on the nature of disorder in the NMe solvate structure.

\section{References}

(1) Nangia, A.; R. Desiraju, G., Pseudopolymorphism: occurrences of hydrogen bonding organic solvents in molecular crystals. Chemical Communications 1999, (7), 605-606.

(2) Mukherjee, A.; Grobelny, P.; Thakur, T. S.; Desiraju, G. R., Polymorphs, Pseudopolymorphs, and Co-Crystals of Orcinol: Exploring the Structural Landscape with High Throughput Crystallography. Crystal Growth \& Design 2011, 11, (6), 2637-2653.

(3) Bajpai, A.; Scott, H. S.; Pham, T.; Chen, K.-J.; Space, B.; Lusi, M.; Perry, M. L.; Zaworotko, M. J., Towards an understanding of the propensity for crystalline hydrate formation by molecular compounds. IUCrJ 2016, 3, (6), 430-439.

(4) Loschen, C.; Klamt, A., Computational Screening of Drug Solvates. Pharmaceutical Research 2016, 33, (11), 2794-2804.

(5) Campeta, A. M.; Chekal, B. P.; Abramov, Y. A.; Meenan, P. A.; Henson, M. J.; Shi, B.; Singer, R. A.; Horspool, K. R., Development of a Targeted Polymorph Screening Approach for a Complex Polymorphic and Highly Solvating API. Journal of Pharmaceutical Sciences 2010, 99, (9), 3874-3886.

(6) Roberts, K. J.; Sherwood, J. N.; Yoon, C. S.; Docherty, R., Understanding the Solvent-Induced Habit Modification of Benzophenone in Terms of Molecular Recognition at the Crystal/Solution Interface. Chemistry of Materials 1994, 6, (8), 1099-1102.

(7) ter Horst, J. H.; Geertman, R. M.; van Rosmalen, G. M., The effect of solvent on crystal morphology. J. Cryst. Growth 2001, 230, (1-2), 277-284.

(8) Hammond, R. B.; Pencheva, K.; Ramachandran, V.; Roberts, K. J., Application of grid-based molecular methods for modeling solvent-dependent crystal growth morphology: Aspirin crystallized from aqueous ethanolic solution. Crystal Growth \& Design 2007, 7, (9), 1571-1574.

(9) Chen, J.; Trout, B. L., Computer-Aided Solvent Selection for Improving the Morphology of Needle-like Crystals: A Case Study of 2,6-Dihydroxybenzoic Acid. Crystal Growth \& Design 2010, 10, (10), 4379-4388.

(10) Hod, I.; Mastai, Y.; Medina, D. D., Effect of solvents on the growth morphology of dl-alanine crystals. CrystEngComm 2011, 13, (2), 502-509.

(11) Li Destri, G.; Marrazzo, A.; Rescifina, A.; Punzo, F., How Molecular Interactions Affect Crystal Morphology: The Case of Haloperidol. Journal of Pharmaceutical Sciences 2011, 100, (11), 4896-4906.

(12) Punzo, F., Unveiling the role of molecular interactions in crystal morphology prediction. Journal of Molecular Structure 2013, 1032, (0), 147-154.

(13) Singh, M. K.; Banerjee, A., Role of Solvent and External Growth Environments to Determine Growth Morphology of Molecular Crystals. Crystal Growth \& Design 2013, 13, (6), 2413-2425.

(14) McCrone, W., Polymorphism. In Physics and Chemistry of the Organic Solid State, Fox, D.; Labes, M. M.; Weissberger, A., Eds. Interscience: New York, 1965; Vol. 11.

(15) MacLeod, C. S.; Muller, F. L., On the Fracture of Pharmaceutical Needle-Shaped Crystals during Pressure Filtration: Case Studies and Mechanistic Understanding. Organic Process Research \& Development 2012, 16, (3), 425-434. 
(16) McArdle, P.; Hu, Y.; Lyons, A.; Dark, R., Predicting and understanding crystal morphology: the morphology of benzoic acid and the polymorphs of sulfathiazole. CrystEngComm 2010, 12, (10), 3119-3125.

(17) Rosbottom, I.; Roberts, K. J.; Docherty, R., The solid state, surface and morphological properties of paminobenzoic acid in terms of the strength and directionality of its intermolecular synthons. CrystEng Comm 2015, 17, (30), 5768-5788.

(18) Walshe, N.; Crushell, M.; Karpinska, J.; Erxleben, A.; McArdle, P., Anisotropic Crystal Growth in Flat and Nonflat Systems: The Important Influence of van der Waals Contact Molecular Stacking on Crystal Growth and Dissolution. Crystal Growth \& Design 2015, 15, (7), 3235-3248.

(19) Aaltonen, J.; Allesø, M.; Mirza, S.; Koradia, V.; Gordon, K. C.; Rantanen, J., Solid form screening - A review. European Journal of Pharmaceutics and Biopharmaceutics 2009, 71, (1), 23-37.

(20) Bauer, J.; Spanton, S.; Henry, R.; Quick, J.; Dziki, W.; Porter, W.; Morris, J., Ritonavir: An Extraordinary Example of Conformational Polymorphism. Pharmaceutical Research 2001, 18, (6), 859-866.

(21) Walker, E. M.; Roberts, K. J.; Maginn, S. J., A Molecular Dynamics Study of Solvent and Impurity Interaction on the Crystal Habit Surfaces of $\varepsilon$-Caprolactam. Langmuir 1998, 14, (19), 5620-5630.

(22) Clydesdale, G.; Hammond, R. B.; Roberts, K. J., Molecular Modeling of Bulk Impurity Segregation and ImpurityMediated Crystal Habit Modification of Naphthalene and Phenanthrene in the Presence of Heteroimpurity Species. The Journal of Physical Chemistry B 2003, 107, (20), 4826-4833.

(23) Kwokal, A.; Nguyen, T. T. H.; Roberts, K. J., Polymorph-Directing Seeding of Entacapone Crystallization in Aqueous/Acetone Solution Using a Self-Assembled Molecular Layer on Au (100). Crystal Growth \& Design 2009, 9, (10), 4324-4334.

(24) Nguyen, T. T. H.; Hammond, R. B.; Roberts, K. J.; Marziano, I.; Nichols, G., Precision measurement of the growth rate and mechanism of ibuprofen $\{001\}$ and $\{011\}$ as a function of crystallization environment. CrystEngComm 2014, 16, (21), 4568-4586.

(25) Toroz, D.; Rosbottom, I.; Turner, T. D.; Corzo, D. M. C.; Hammond, R. B.; Lai, X.; Roberts, K. J., Towards an understanding of the nucleation of alpha-para amino benzoic acid from ethanolic solutions: a multi-scale approach. Faraday Discussions 2015, 179, (0), 79-114.

(26) Li, J.; Tilbury, C. J.; Joswiak, M. N.; Peters, B.; Doherty, M. F., Rate Expressions for Kink Attachment and Detachment During Crystal Growth. Crystal Growth \& Design 2016, 16, (6), 3313-3322.

(27) Roberts, K. J.; Hammond, R. B.; Ramachandran, V.; Docherty, R., Synthonic engineering: from molecular and crystallographic structure to the rational design of pharmaceutical solid dosage forms. In Computational Approaches in Pharmaceutical Solid State Chemistry Abramov, Y., Ed. Wiley: New Jersey, USA, 2016.

(28) Tilbury, C. J.; Green, D. A.; Marshall, W. J.; Doherty, M. F., Predicting the Effect of Solvent on the Crystal Habit of Small Organic Molecules. Crystal Growth \& Design 2016, 16, (5), 2590-2604.

(29) McArdle, P., Oscail, a program package for small-molecule single-crystal crystallography with crystal morphology prediction and molecular modelling. Journal of Applied Crystallography 2017, 50, (1), 320-326.

(30) Gron, H.; Borissova, A.; Roberts, K. J., In-Process ATR-FTIR Spectroscopy for Closed-Loop Supersaturation Control of a Batch Crystallizer Producing Monosodium Glutamate Crystals of Defined Size. Industrial \& Engineering Chemistry Research 2003, 42, (1), 198-206.

(31) Ma, C. Y.; Wang, X. Z., Model identification of crystal facet growth kinetics in morphological population balance modeling of l-glutamic acid crystallization and experimental validation. Chemical Engineering Science 2012, $70,22-30$.

(32) Duffy, D.; Barrett, M.; Glennon, B., Novel, Calibration-Free Strategies for Supersaturation Control in Antisolvent Crystallization Processes. Crystal Growth \& Design 2013, 13, (8), 3321-3332.

(33) Constance, E. N.; Mohammed, M.; Mojibola, A.; Egiefameh, M.; Daodu, O.; Clement, T.; Ogundolie, T.; Nwawulu, C.; Aslan, K., Effect of additives on the crystal morphology of amino acids: A theoretical and experimental study. J. Phys. Chem. C 2016, 120, (27), 14749-14757.

(34) Flaten, E. M.; Seiersten, M.; Andreassen, J. P., Polymorphism and morphology of calcium carbonate precipitated in mixed solvents of ethylene glycol and water. J. Cryst. Growth 2009, 311, (13), 3533-3538.

(35) Zhang, L.; Yue, L. H.; Wang, F.; Wang, Q., Divisive effect of alcohol-water mixed solvents on growth morphology of calcium carbonate crystals. J. Phys. Chem. B 2008, 112, (34), 10668-10674.

(36) Clydesdale, G.; Roberts, K. J.; Docherty, R., Modelling the morphology of molecular crystals in the presence of disruptive tailor-made additives. J. Cryst. Growth 1994, 135, (1), 331-340.

(37) Clydesdale, G.; Roberts, K. J.; Lewtas, K.; Docherty, R., Modelling the morphology of molecular crystals in the presence of blocking tailor-made additives. J. Cryst. Growth 1994, 141, (3-4), 443-450.

(38) Kuvadia, Z. B.; Doherty, M. F., Effect of Structurally Similar Additives on Crystal Habit of Organic Molecular Crystals at Low Supersaturation. Crystal Growth \& Design 2013, 13, (4), 1412-1428.

(39) Lovette, M. A.; Doherty, M. F., Needle-Shaped Crystals: Causality and Solvent Selection Guidance Based on Periodic Bond Chains. Crystal Growth \& Design 2013, 13, (8), 3341-3352. 
(40) Vinodhini, K.; Srinivasan, K., The role of a mixture of DMSO: water in the crystallization of alpha-lactose monohydrate (alpha-LM) single crystals with desired morphology. CrystEngComm 2015, 17, (33), 6376-6383.

(41) Cook, J. L.; Hunter, C. A.; Low, C. M. R.; Perez-Velasco, A.; Vinter, J. G., Preferential Solvation and Htdrogen Bonding in Mixed Solvents. Angew. Chem. Int. Ed. 2008, 47, 6275-7277.

(42) Prausnitz, J. M., Molecular Thermodynamics of Fluid-Phase Equilibria. ed.; Prentice-Hall: 1969.

(43) Roberts, K. J.; Hammond, R. B.; Ramachandran, V.; Docherty, R., Synthonic engineering: from molecular and crystallographic structure to the rational design of pharmaceutical solid dosage forms. In Computational Approaches in Pharmaceutical Solid State Chemistry, Abramov, Y. A., Ed. Wiley: New Jersey, USA, 2016.

(44) Moldovan, A. A.; Rosbottom, I.; Ramachandran, V.; Pask, C. M.; Olomukhoro, O.; Roberts, K. J., Crystallographic Structure, Intermolecular Packing Energetics, Crystal Morphology and Surface Chemistry of Salmeterol Xinafoate (Form I). Journal of Pharmaceutical Sciences 106, (3), 882-891.

(45) Svard, M.; Nordstrom, F. L.; Hoffmann, E.-M.; Aziz, B.; Rasmuson, A. C., Thermodynamics and nucleation of the enantiotropic compound p-aaminobenzoic acid. CrystEngComm 2013.

(46) Hao, H. X.; Barrett, M.; Hu, Y.; Su, W. Y.; Ferguson, S.; Wood, B.; Glennon, B., The Use of in Situ Tools To Monitor the Enantiotropic Transformation of p-Aminobenzoic Acid Polymorphs. Organic Process Research \& Development 2012, 16, (1), 35-41.

(47) Gracin, S.; Rasmuson, ^. C., Polymorphism and Crystallization of p-Aminobenzoic Acid. Crystal Growth \& Design 2004, 4, (5), 1013-1023.

(48) Black, J. F. B.; Davey, R. J.; Gowers, R. J.; Yeoh, A., Ostwald's rule and enantiotropy: polymorph appearance in the crystallisation of p-aminobenzoic acid. CrystEng Comm 2015, 17, (28), 5139-5142.

(49) Sullivan, R. A.; Davey, R. J., Concerning the Crystal Morphologies of the alpha and beta polymorphs of paminobenzoic acid. CrystEngComm 2015, 17, 1015-1023.

(50) Toroz, D.; Rosbottom, I.; Turner, T.; Corzo, D. M. C.; Hammond, R. B.; Lai, X.; Roberts, K. J., Towards An Understanding of the Nucleation of Para Amino Benzoic Acid: A Multi Scale Approach. Faraday Discussions 2015, 179, 79-114.

(51) Prausnitz, J. M., Molecular Thermodynamics of Fluid Phase Equilibria. ed.; 1969.

(52) Turner, T. D.; Corzo, D. M. C.; Toroz, D.; Curtis, A.; Dos Santos, M. M.; Hammond, R. B.; Lai, X.; Roberts, K. J., The influence of solution environment on the nucleation kinetics and crystallisability of para-aminobenzoic acid. Physical Chemistry Chemical Physics 2016, 18, (39), 27507-27520.

(53) http://www.julabo.com/en.

(54) http://www.panalytical.com/Home.htm.

(55) Cosier, J.; Glazer, A. M., A nitrogen-gas-stream cryostat for general X-ray diffraction studies. Journal of Applied Crystallography 1986, 19, 105-107.

(56) Diffraction, R. O., CrysAlisPRO. In ed.; Rigaku Oxford Diffraction: England, 2015.

(57) Clark, R. C.; Reid, J. S., The analytical calculation of absorption in multifaceted crystals. Acta Crystallographica Section A Foundations of Crystallography 1995, 51, 887-897.

(58) Sheldrick, G. M., SHELXS97, Program for the Solution of Crystal Structures. A short history of SHELX 2008, 64, $112-122$.

(59) Sheldrick, G. M., Crystal structure refinement with SHELXL. Acta Crystallographica Section C: Structural ... 2015.

(60) Dolomanov, O. V.; Bourhis, L. J.; Gildea, R. J.; Howard, J. A. K.; Puschmann, H., OLEX2: A complete structure solution, refinement and analysis program. Journal of Applied Crystallography 2009, 42, 339-341.

(61) Bladon, P.; Gorton, J.; Hammond, R. B., Molecular Modelling: Computational Chemistry Demystified. ed.; RSC: Cambridge, UK, 2011.

(62) Bladon, P. Interchem, Glasgow, UK, 2013.

(63) Clydesdale, G.; Docherty, R.; Roberts, K. J., HABIT - a program for predicting the morphology of molecular crystals. Comput Phys Commun 1991, 64, (2), 311-328.

(64) Momany, F. A.; Carruthers, L. M.; McGuire, R. F.; Scheraga, H. A., Intermolecular potentials from crystal data. III. Determination of empirical potentials and application to the packing configurations and lattice energies in crystals of hydrocarbons, carboxylic acids, amines, and amides. The Journal of Physical Chemistry 1974, 78, (16), 1595-1620.

(65) Dewar, M. J. S.; Zoebisch, E. G.; Healy, E. F.; Stewart, J. J. P., Development and use of quantum mechanical molecular models. 76. AM1: a new general purpose quantum mechanical molecular model. Journal of the American Chemical Society 1985, 107, (13), 3902-3909.

(66) Stewart, J. J. P., MOSOL, MOPAC for Solid-State Physics. Quant. Chem. Prog. Exchange 1985, 5, 62-63.

(67) Docherty, R.; Roberts, K. J., Modeling The Morphology Of Molecular-Crystals - Application To Anthracene, Biphenyl And Beta-Succinic Acid. J Cryst Growth 1988, 88, (2), 159-168. 
(68) Docherty, R.; Clydesdale, G.; Roberts, K. J.; Bennema, P., Application Of Bravais-Friedel-Donnay-Harker, Attachment Energy And Ising-Models To Predicting And Understanding The Morphology Of Molecular-Crystals. J. Phys. D-Appl. Phys. 1991, 24, (2), 89-99.

(69) Roberts, K. J.; Docherty, R.; Bennema, P.; Jetten, L., The Importance Of Considering Growth-Induced Conformational Change In Predicting The Morphology Of Benzophenone. J. Phys. D-Appl. Phys. 1993, 26, (8B), B7-B21.

(70) Clydesdale, G.; Roberts, K. J.; Telfer, G. B.; Saunders, V. R.; Pugh, D.; Jackson, R. A.; Meenan, P., Prediction of the Polar Morphology of Sodium Chlorate Using a Surface-Specific Attachment Energy Model. The Journal of Physical Chemistry B 1998, 102, (36), 7044-7049.

(71) Hammond, R. B.; Ma, C. Y.; Roberts, K. J.; Ghi, P. Y.; Harris, R. K., Application of Systematic Search Methods to Studies of the Structures of Urea-Dihydroxy Benzene Cocrystals. The Journal of Physical Chemistry B 2003, 107, (42), 11820-11826.

(72) Hammond, R. B.; Hashim, R. S.; Ma, C.; Roberts, K. J., Grid-based molecular modeling for pharmaceutical salt screening: Case example of 3,4,6,7,8,9-hexahydro-2H-pyrimido (1,2-a) pyrimidinium acetate. Journal of Pharmaceutical Sciences 2006, 95, (11), 2361-2372.

(73) Hammond, R. B.; Pencheva, K.; Roberts, K. J., Molecular modeling of crystal-crystal interactions between the alpha- and beta-polymorphic forms of L-glutamic acid using grid-based methods. Crystal Growth \& Design 2007, 7, (5), $875-884$.

(74) Hammond, R. B.; Pencheva, K.; Ramachandran, V.; Roberts, K. J., Application of grid-based molecular methods for modeling solvent-dependent crystal growth morphology: Aspirin crystallized from aqueous ethanolic solution. Cryst Grow Des 2007, 7, (9), 1571-1574.

(75) Ramachandran, V.; Murnane, D.; Hammond, R. B.; Pickering, J.; Roberts, K. J.; Soufian, M.; Forbes, B.; Jaffari, S.; Martin, G. P.; Collins, E.; Pencheva, K., Formulation Pre-screening of Inhalation Powders Using Computational AtomAtom Systematic Search Method. Molecular Pharmaceutics 2014, 12, (1), 18-33.

(76) Toroz, D.; Rosbottom, I.; Turner, T. D.; Corzo, D. M. C.; Hammond, R. B.; Lai, X.; Roberts, K. J., Towards an understanding of the nucleation of alpha-para amino benzoic acid from ethanolic solutions: a multi-scale approach. Faraday Discussions 2015, 179, 79-114.

(77) Killean, R. C. G.; Tollin, P.; Watson, D. G.; Young, D. W., Twinning in p-aminobenzoic acid. Acta Crystallographica 1965, 19, (3), 482-483.

(78) Sullivan, R. A.; Davey, R. J.; Sadiq, G.; Dent, G.; Back, K. R.; ter Horst, J. H.; Toroz, D.; Hammond, R. B., Revealing the Roles of Desolvation and Molecular Self-Assembly in Crystal Nucleation from Solution: Benzoic and pAminobenzoic Acids. Crystal Growth \& Design 2014, 14, (5), 2689-2696.

(79) Rosbottom, I. The Influence of Molecular Interactions and Aggregation on the Polymorphism, Crystal Growth and Morphology of Para Amino Benzoic Acid University of Leeds, Leeds, 2015.

(80) Turner, T. D. Molecular self-assembly, nucleation kinetics and cluster formation associated with solution crystallisation. University of Leeds, Leeds, UK, 2015. 